Magnetic Separation News, 1986, Vol. 2, pp. 51-68

$0731-3632 / 86 / 0202-0051 \$ 25.00 / 0$

(C) 1986 Gordon and Breach, Science Publishers, Inc.

Printed in the United Kingdom

\title{
APPLICATION OF HIGH-GRADIENT MAGNETIC \\ SEPARATION
}

J. SVOBODA

Council for Mineral Technology, Randburg, South Africa

Abstract some problems of practical
applications of magnetic separation,
primarily in mineral industry, are
outlined. The work shows that current
theoretical models are inadequate in
accounting for the magnetic separation of
valuable mineral components. The negative
impact of a lack of cost-effective
separators in the mining industry is
discussed.

\section{INTRODUCTION}

In recent years, magnetic separation, which is one of the oldest techniques for the treatment of minerals, has undergone considerable progress in various branches of industry. High-gradient magnetic separation (HGMS) has become a powerful technique for the handling of finely dispersed weakly magnetic particles but, despite its merits, it has been used on an industrial scale in only a limited number of applications, primarily in the kaolin industry and in water filtration. 
The beneficiation of minerals represents the largest potential field of application for HGMS, since magnetic separation can produce high-quality concentrates from a large variety of minerals. However, the large-scale application of HGMS to the treatment of minerals is restricted by two basic factors :

(a) the lack of a cost-effective continuous separator that can be used for the whole range of minerals, and

(b) an inadequate understanding of the fundamental principles of HGMS as applied to complex systems of minerals, which causes difficulties when HGMS is incorporated into circuits for the beneficiation of minerals.

In contrast to the practical problem in HGMS, those in flotation are well understood, although the mechanism of flotation is inherently more complicated. Flotation benefited from the increased interest shown by scientists in mineral processing in the 1930s, and established itself as a powerful technique for the recovery of valuable minerals, thus gaining the confidence of the mining industry. Magnetic separation in its simplest form is also an inherent part of flowsheets, but HGMS is limited largely to fairly unprofitable applications in the filtration of wastes.

The responsible plant engineer trying to incorporate HGMS into a flowsheet is faced with the almost insurmountable problem of finding a sufficiently simple and clear algorithm that will enable him to apply HGMS to his circuit. Usually, he 
has to resort to a trial-an-error approach and to the classical handbooks and papers on magnetic separation.1-3 current theoretical and experimental investigation into the fundamental principles of HGMS is limited largely to diluted systems of reasonably well-defined compositions, and is aimed at the acquisition of a better understanding of the process of particle capture on a matrix as a function of particle size, magnetic induction, flow velocity, and matrix configuration. In most instances, the conclusions drawn from these studies are scarcely relevant to practical problems, and can often be misleading.

surprisingly enough, very little attention has been paid to the effects of some of the fundamental parameters of HGMS, namely the size and magnetization of the matrix, the viscosity of a slurry, and the magnetic susceptibility of the particles. Very little is known about the cooperative phenomena in mineral slurries and the effect of irregular particle shape, although these factors may substantially affect HGMS.

The role of the basic parameters is well understood in magnetic filtration, where the 'brute force' approach is usually employed in efforts to attain a high efficiency of capture. However, the role of these parameters changes considerably for complex systems in which several mineral species are present and in which the magnetic fraction is often the valuable one. It is commonly believed that the performance of a separation device measured in terms of recovery depends on the ratio of the magnetic force acting on the particles to the sum of the 
competing forces ${ }^{4}$. Although this simple rule applies well to magnetic filtration, it does not apply to the beneficiation of complex ores where the efficiency and the selectivity of capture must be maximized simultaneously. Moreover, such mineral systems usually comprise a wide range of particle sizes and magnetic susceptibilities, and it is clear that, in the application of magnetic separation to such systems, any approach in which the magnetic force or the magnetic velocity was maximized would be invalid. The minerals engineer therefore has to resort to a trial-and-error approach in determining the operating conditions.

This paper briefly discusses several features of HGMS that are of practical importance but are usually overlooked in current theoretical treatises on HGMS.

\section{MAGNETIC FORCE}

The magnetic force acting on a small magnetic particle is given by

$$
\vec{F}_{M}=x V_{p} \cdot \operatorname{grad}\left(\frac{1}{2} H B_{0}\right)
$$

where $H$ is the magnitude of the magnetic field, $B_{0}$ is the magnitude of applied magnetic-flux density, $v_{p}$ is the volume of the particle, and $\chi$ is the difference between the volume magnetic susceptibility of the particle and of the carrier fluid.

The force is usually considered ${ }^{5}$ to be a product 
of $\chi \nabla_{p}$, which describes the physical properties of the particles, and of the magnetic force density:

$$
\vec{f}_{M}=\operatorname{grad}\left(\frac{1}{2} H B_{0}\right)
$$

According to Gerber ${ }^{5}$, this is the principal single parameter that can be attributed to a magnetic separator and utilized for its classification.

However, this approach treats the magnetic field and the gradient of the magnetic field in a symmetrical way, although their effects on the physical properties of the mineral particles and on the selectivity of the process are substantially different. Most minerals containing a parasitic ferromagnetic component exhibit magnetic susceptibilities that are dependent on the magnetic field. The effect of a variable magnetic susceptibility on HGMS, which had been demonstrated experimentally $y^{6}$, was incorporated into a model of HGMS by Nesset and Finch $^{7}$. The magnetic properties of the mineral particles therefore depend on the operating value of the magnetic induction, and not on the gradient of the field.

Likewise, a uniform magnetic field induces the agglomeration of mineral particles, which substantially affects the recovery and the grade of the magnetic fraction. On the one hand, bigger clusters of magnetic particles have a higher probability of being captured onto a matrix, thus enhancing the recovery; on the other, gangue particles entrained in these agglomerates impair the grade. At high values of magnetic field, even the 
gangue particles can agglomerate and can be captured magnetically or mechanically, by the matrix, again at the expense of the grade of the magnetic fraction.

It is therefore obvious that a system of mineral particles behaves differently at different strengths of the background magnetic field but at the same value of the magnetic-force density given by equation (2). Hence, the magnetic-force density is inappropriate as a classification parameter.

\section{MATRIX MATCHING}

The importance of the choice of a suitable matrix for the concentration of valuable mineral components cannot be over-emphasized. The geometry and size of a matrix play a decisive role in the achievement of optimum recovery and grade of concentrate and determine the matrix loading, a crucial factor in large-scale magnetic separations. Oberteuffer ${ }^{8}$ and Aharoni' show theoretically that the magnetic force achieves a maximum when the ratio of the radius of the matrix collector to the radius of the particle $(a / b)$ is approximately equal to unity. The graph for this maximum was found to be fairly flat, and it was confirmed experimentally $y^{0}$ that, in the range of (a/b) from 1 to 10, the efficiency of capture shows little change. Other work has indicated ${ }^{1}$ that the optimum ratio of collector size to particle size is not constant, so that the matching of particle size with collector size8,9 is somewhat complicated. Also, the theoretical analysis'2 of the spatial distribution of magnetic force implies that the 
optimum value for the ratio of matrix size to particle size lies between 5 and 10 , being a function of magnetic induction, particle size, and the type of matrix used.

Although the results of the single-collectorsingle-particle studies contribute towards a better understanding of the dynamics of a magnetizable particle in the vicinity of an isolated magnetized collector, their validity for a real matrix is very limited.

Attempts to maximize the ratio of traction magnetic force to drag force usually result in the capture of a particle by a mechanical straining mechanism. If $a / b<10$, the straining mechanism is dominant and leads to the formation of a mat of deposit on the surface of the matrix. This layer of deposit causes a high resistance to the flow, increases the losses of the non-magnetic product in those applications where magnetic impurities are removed, or severely impairs the grade of the magnetic concentrate in those applications where the valuable minerals are concentrated into the magnetic fraction. Naturally, the straining mechanism is undesirable and should be avoided in the design of magnetic separators. For straining to be minimized, $a / b$ should be larger than 10, but should preferably range from 100 to 500 .

In the application of HGMS to the beneficiation of minerals, the matrix size must be matched to the particle size in such a way that recovery and grade are maximized simultaneously. The optimum matrix size thus becomes a function of the size distribution and the composition of the ore. The 
usual practice is a tedious trial-and-error search for the best shape and size of matrix in which several fundamental rules have to be kept in mind. With decreasing size of the matrix element, the recovery of the magnetic fraction rises as the grade of the concentrate decreases. With very fine matrices, the recoveries become high and the grades so low that the grade of the 'magnetic concentrate' may not differ very much from that of the feed. A limited number of publications dealing with the selection of matrices for the magnetic separation of finely ground weakly magnetic ores give the optimum size of the matrix element as ranging from 1 to

$10 \mathrm{~mm}$ for the particle-size distributions $80 \%-37$

$\mu \mathrm{m}^{13}, 90 \%-74 \mu \mathrm{m}^{14,15}, 43 \%-38 \mu \mathrm{m}^{16}$ and $50 \%-22$

$\mu \mathrm{m}^{17}$. In these instances, the ratio of the collector size to the particle size amounts to about 300 , i.e. two orders of magnitude larger than the optimum ratio. Very similar types of matrices were found to give the best results in the removal of pyrite from $\operatorname{coal}^{18}$, with the magnetic susceptibility an order of magnitude lower and the particle size an order of magnitude higher than in the instance mentioned above. If fine matrices with a ratio of fibre to particle size of about 10 are used, the grade of the magnetic concentrate becomes unpractically low and approaches that of the feed. At the same time, the matrix becomes excessively susceptible to clogging by oversize grains, ferromagnetic impurities, and other foreign bodies that are always present in ore pulps.

Observation of the basic rules of matrix optimization fails even in simple filtration 
experiments $^{19}$. For the filtration of ferric hydroxide flocs with a mean diameter of $3 \mu \mathrm{m}$, a ball matrix was as effective as a fine-mesh matrix, the filaments of which have a diameter approximately 20 times smaller than that of the balls.

\section{THE EFFECTS OF SATURATION MAGNETIZATION} AND VISCOSITY

As follows from a simple model of a balance of forces $^{8}$ or from Watson's model ${ }^{20}$, the probability of capture is directly proportional to the magnetization of the matrix material, and inversely proportional to the viscosity of the fluid. Limited published experimental data indicate substantial deviations from this outcome of the simple theory.

It has been shown ${ }^{15}$ that, at the intermediate magnetic field, a paramagnetic steel matrix with a magnetization of $0,1 T$ is only slightly less efficient than a standard mild-steel matrix with a magnetization of $2 T$ and, when the external magnetic field is increased, the performance of both types of steels will be equal.

A detailed investigation of the effect of magnetization on the beneficiation of iron ore 17 has shown that, for a wide range of steels with magnetization values varying from 0,5 to $2,1 \mathrm{~T}$, the recovery of iron is independent of the magnetization. Also, the quality of the magnetic fraction does not depend on the magnetization, and this is valid for an even wider interval of magnetization values ${ }^{15}$.

Very similar rules were found to apply to the 
purification of kaolin'1. Amorphous ferromagnetic fibres with a saturation magnetization of $1,1 \mathrm{~T}$ performed better under identical conditions than ordinary stainless-steel wool with a saturation magnetization of $2,1 \mathrm{~T}$.

Recent theoretical analysis 22 shows that the behaviour of a real magnetic separator is determined largely by the geometry of the fluid through the matrix, rather than by the magnetic properties of the matrix.

The question of the effect of magnetization is important, not only from the point of view of the theoretical models, but has some serious practical consequences. Saturation magnetization of the matrices in commercial separators is known only in rare instances, which also applies when the worn-out matrix must be replaced. The operator then tends to attribute the inadequate metallurgical performance of a separator to the inferior magnetic properties of a matrix, although the effect of saturation magnetization would often be of a minor character, if any.

Similar reservations apply to the role of viscosity. Dobby and Finch $^{23}$ showed that viscosity is of minor importance in the capture of mineral particles by HGMS. The use of liquid nitrogen as a carrier fluid had very little effect on the upgrading of a coal, despite its advantage in increasing the susceptibility of the pyrite ${ }^{4}$.

\section{THE LIMITING PARTICLE SIZE}

Theoretical studies of idealized simple models have led to an understanding of the basic mechanisms 
involved in the capture process, and usually give acceptable agreement with experimental data on the removal of solid particles from simple, artificially prepared slurries or from diluted, single-component industrial suspensions. For these models to be useful in the selection of the initial operating conditions for the separation of industrially treated mineral systems, they have to give an answer to the fundamental question of how fine a particle can be recovered by HGMS. This question is obviously very relevant to the engineer, but is not clearly addressed in the literature.

The oberteuffer model ${ }^{8}$ gives much higher values for the limiting particle size than the other models, and the agreement with experimental data is poor. Although watson's ${ }^{20}$ and Nesset and Finch's ${ }^{7}$ models give lower values for the limiting particle size, these values are still an order of magnitude greater than those obtained by the present author from the experimental data for the magnetic separation of iron ores ${ }^{25}$.

Hencl et al. recognized that a modified physical picture was needed ${ }^{26}$. They argued that the erosion force was the dominant competing force in a real matrix, and obtained better agreement between theory and experiment.

The inadequacy of the current theoretical models to predict the smallest particle to be captured in HGMS is demonstrated by the recovery of uranium and gold from leach residues. The models predict the limiting particle diameter to be about $60 \mu \mathrm{m}$ for a ball matrix, although the experimental recovery of uranium and gold was 70 percent with very high 
grades from a sample in which the particle-size distribution was $55 \%-22 \mu \mathrm{m}$ and $20 \%-5 \mathrm{~m}^{17}$. $\mathrm{High}$ recoveries of uranium and gold were obtained even under such mild conditions as $V_{M} / V_{0}=0,2$ or $N_{L}=$ 10, where $V_{M}$ is the usual magnetic velocity ${ }^{20}, V_{0}$ is the flow velocity, and $N_{L}$ is the loading factor ${ }^{7}$. Recoveries under the above conditions did not differ from the results obtained at $V_{M} / V_{0}=50$ or $N_{L}=800$, although the theory predicts a strong capture when $V_{M} / V_{0} \geq 1$ or $N_{L} \geq 25$.

It is clear, therefore, that there is no reliable means by which the operating conditions in the separation of valuable minerals can be predicted, and that the process of selective separation is much more complex than the mere removal of unwanted impurities.

The failure of the models to predict the limiting particle size correctly is not surprising. The models are based on simplified conditions that are rarely met in practice and they neglect a number of interactions that become important in the concentration of industrial slurries. For instance, co-operative phenomena like homo- and heterocoagulation substantially affect the performance of a magnetic separator, as has been shown

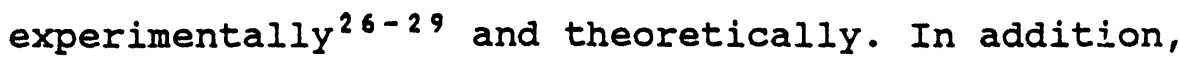
the 'piggy-back' magnetic separation contributes significantly to the failure of simple models when they are applied to mineral pulps.

\section{MAGNETIC SEPARATORS}

As was stressed in the beginning, one of the reasons 
why HGMS does not fulfil its promise is that there is a lack of reliable, cost-effective magnetic separators. The relatively cheap solenoid cyclic separators are suitable for only a limited number of applications. The kaolin industry uses such separators extensively, but their use in waste-water treatment is too expensive, an important factor in a time of recession. Their use in the filtration of cooling water in nuclear-power plants is at present too costly owing to the additional welding, supplementary installations, and safety precautions required.

The minerals industry, which is potentially the largest user of HGMS, requires large continuous units. The original carousel separators, which employ a conventional iron-yoke magnetic circuit, generate only a modest magnetic induction in a small working volume and have a limited capacity. It is unlikely that larger, more powerful machines can be built. This present two distinct drawbacks: the cost-effectiveness cannot be improved substantially, and any large-capacity operation could require several dozen industrial machines ${ }^{30}$. Problems associated with the distribution of the feed, the pumping of tailings and concentrates, maintenance, and the like become enormous.

The problem of large-capacity machines has partially been solved. Iron-clad solenoid continuous machines can be scaled-up easily, a modest input power still being preserved to generate high magnetic fields. The SALA carouse 31,32 for HGMS and the drum high-gradient magnetic separator ${ }^{14}$ are units that could find wide application in all 
branches of the minerals industry. As shown by Roux et $a l^{33}$ and Forsberg et $a l^{34}$, such separators have a distinct advantage over a whole range of other machines, although factors such as the extent of matrix blockage on these machines are still unknown and their cost-effectivness is still low.

The Council for Mineral Technology (Mintek) is currently commissioning a large-capacity continuous machine $e^{5}$ that has a cost-effectiveness up to 5 times higher than that of the above-mentioned separators. The unit cost of the machine will make it possible for the flowsheet to be simplified, which will mean in turn that the overall capital costs and the operating costs will be lower than those for current HGMS installations.

\section{CONCLUSIONS}

on the whole, the theoretical models for magnetic separation satisfactorily describe the experiments on diluted suspension in which the removal of magnetic impurities is the primary task. In morecomplex instances, for example in the magnetic separation of valuable mineral components, the theory is inadequate. In order to win the confidence of the mining industry in HGMS, less-complex and more practically orientated theoretical description is needed, and more-extensive contact between academic and industrial circles must be established. owing to its tremendous potential, HGMS deserves attention that will lead to major advances of the technology within a reasonable time. 


\section{REFERENCES}

1. A.F. TAGGART, Elements of ore dressing (John Wiley, New York, 1951).

2. V.G. DERKAC, The special methods of the beneficiation of ores, Nedra, Moscow, 1966.

3. J.E. LAVWER, D.M. HOPSTOCK, Miner. SCi. Engng., 6 (1974) p. 154 .

4. M.R. PARKER, Recent developments in high-field magnetic separation., Proceedings of the International symposium on Electrical and Magnetic Separation and Filtration Technology, Antwerp, 1984.

5. R. GERBER and R.R. BIRSS, High gradient magnetic separation (John Wiley \& sons Ltd., Chichester, 1983).

6. J.M. PASTRANA and D.M. HOPSTOCK, Trans. SOC. Min.Eng., AIME 262 (1977) p.1.

7. J.E. NESSET and J.A. FINCH, A static model of HGMS based on forces within the boundary layer, Proceedings International conference on Industrial Applications of Magnetic Separation, IEEE Publ. no. $78 \mathrm{CH}, 1447-2$ (1979), p. 188.

8. J.A. OBERTEUFFER, IEEE Trans. On Mags., MAG-10 (1977), p. 223 . 
9. A. AHARONI, IEEE Trans. on Mags., MAF-12 (1976), P. 237 .

10. H.K. COLLAN, et al., IEEE Trans. on Mags. MAG17 (1978), p. 398 .

11. C.J. CLARKSON, and D.R. KELLAND, IEEE Trans. on Mags., MAG-14 (1978), P. 97.

12. E. MADAI, Aufbereitungs-Tech, 24 (1983), p. 99.

13. D.M. HOPSTOCK, and A.F. COLOMBO, Processing finely ground oxidized taconites by WHIMS, Proceedings of Conference on Fines Particles Processing, (Las Vegas, 1980), p. 1242.

14. V. HENCL. Rudy, 30 (1982), p. 41.

15. J. SVOBODA, Int. J. of Miner. Process., 8 (1981), p. 165

16. I.J. CORRANS, and J. LEVIN, J.S. Afr. Inst. Min. Metall., 29 (1979), p.210.

17. J. SVOBODA, The selection of a matrix for the recovery of uranium by WHIMS. Mintek Report M204 (1985)

18. H. DÖRR, F.P. MONOSTORY, and M. SEIDEL, Gluckauf, 117 (1981), p.11. 
19. J.A. WILIIAMS. et al., Nuclear technology, $\underline{52}$ (1981), p. 284 .

20. J.H.P. WATSON, J. Appl. Phys., 44 (1973), p. 4209 .

21. Y. YING-SHAN, et al., IEEE Trans. on Mags., MAG-16 (1980), p. 934 .

22. J.H.P. WATSON, and S.J.P. WATSON, IEEE Trans. on Mags., MAG-19 (1983), p. 2698.

23. G. DOBBY, and J.A. FINCH, Powder Technol., 17 (1977), p. 73 .

24. J.I. DIJKHUIS, and C.B.W. KERKDIJK, IEEE Trans. on Mags., MAG-17 (1981), p. 1503.

25. J. SVOBODA, Rudy, 29 (1981), p. 133 .

26. V. HENCL, K. JAHODA, and E. MADAI, Analysis of magnetic and hydraulic forces in an oriented real matrix of HGMS, Digest of the Intermag Conference (Hamburg, 1984).

27. J. SVOBODA, Aufbereitungs-Tech., 24 (1983), p. 520 .

28. P. PARSONAGE, Trans. Inst. Min. Metall., C93 $(1984)$, p. C37.

29. J. SVOBODA, I.J. CORRANS, and M. SPITZE, Int. J. Miner. Process. 17 (1986), p. 83 . 
30. I.J. CORRANS, W.A. GILBERT, K.S. IIDDELI, and R.C. DUNNE, J.S. Afr. Inst. Min. Metall., 84 $(1984)$, p. 57 .

31. J.A. OBERTEUFFER, Recent advances in HGMS, Proceedings of Conference on Fine Particles Processing. (Las Vegas, 1980) P. 1178).

32. B. ANDEN, et al., Min. Eng. (N.Y.) 34 (1982), p. 694 .

33. E.H. ROUX, J.G. GOODEY, E.F. WEPENER, and K.R. HODIERNE, Industrial scale dry beneficiation of phosphate-bearing pyroxenite ore, Mintek 50: International Conference on Recent Advances in Mineral Science and Technology (Sandton, 1984).

34. K.S.E. FORSSBERG, and N.R. KOSTKEVICIUS, Erzmetal1, 35 (1982), p. 285 .

35. I.J. CORRANS, and J. SVOBODA, S.A. Patent 83/3323 (Appl. 1983). 